\title{
多羧基咪唑离子液体的酸性表征
}

\author{
耿卫国李雪辉* 王乐夫段红丽潘微平 \\ (华南理工大学化工与能源学院, 广东省绿色化学产品技术重点实验室, 广州 510640 )
}

\begin{abstract}
摘要 通过电位滴定法测得多羧基咪唑离子液体的离解常数 $\mathrm{p} K_{\mathrm{a}}$ 值 $\left(25^{\circ} \mathrm{C}\right)$ 在 $1.43 \sim 1.92$ 范围内, 说明多羧基咪唑 离子液体酸性接近乙二酸, 比丁二酸以及乙酸强. 由于多羒基咪唑离子液体结构中咪夾环具有较强的吸电子诱 导效应, 使多羧基咪唑离子液体具有中等强度的酸性. 三羊基咪唑离子液体酸性比含相同阴离子的二羧基咪唑 离子液体酸性强. 阳离子相同、阴离子不同的多羧基咪唑离子夜体酸性强弱顺序为: $\mathrm{HSO}_{4}^{-}>\mathrm{NO}_{3}^{-}>\mathrm{PF}_{6}^{-}>\mathrm{H}_{2} \mathrm{PO}_{4}^{-}>$ $\mathrm{Cl}^{-} 、 \mathrm{Br}^{-}>\mathrm{CF}_{3} \mathrm{CO}_{2}^{-}>\mathrm{BF}_{4}^{-}>\mathrm{CF}_{3} \mathrm{SO}_{3}^{-}$. 同时, 吡啶红外光谱探针法研究表明, 多羧基咪唑离子液体具有Brønsted酸性.
\end{abstract}

关键词: 离子液体, 多羒基, 酸性表征

中图分类号: $\mathrm{O} 645$

\section{Acidic Characterization of Polycarboxyl Imidazolium Ionic Liquids}

\author{
GENG, Wei-Guo LI, Xue-Hui * WANG, Le-Fu DUAN, Hong-Li PAN, Wei-Ping \\ (The Guangdong Provincial Laboratory of Green Chemical Technology, School of Chemical and Energy Engineering, \\ South China University of Technology, Guangzhou 510640, P. R. China)
}

\begin{abstract}
The acid-dissociation constant $\mathrm{p} K_{\mathrm{a}}$ of different polycarboxyl imidazolium ionic liquids (ILs) has been determined by potentiometric titration method at $25{ }^{\circ} \mathrm{C}$, and the values are in the range of 1.43 to 1.92 , which are close to that of oxalic acid, but much smaller than those of succinic acid and acetic acid. The stronger acidity of polycarboxyl imidazolium ILs results from the strong electron withdrawing effect of positively charged imidazolium ring. It is also demonstrated that the acidity of the ILs is enhanced as the number of carboxylic groups appended to cations increases from two to three. The acidity of the ILs also depends on properties of the anions, and decreases in the order $\mathrm{HSO}_{4}^{-}>$ $\mathrm{NO}_{3}^{-}>\mathrm{PF}_{6}^{-}>\mathrm{H}_{2} \mathrm{PO}_{4}^{-}>\mathrm{Cl}^{-} 、 \mathrm{Br}^{-}>\mathrm{CF}_{3} \mathrm{CO}_{2}^{-}>\mathrm{BF}_{4}^{-}>\mathrm{CF}_{3} \mathrm{SO}_{3}^{-}$. The Brønsted acidity of the polycarboxyl imidazolium ILs has also been studied by IR technique using pyridine as the spectroscopic probe.
\end{abstract}

Keywords: Ionic liquid, Polycarboxylic group, Acidic characterization

近年来, 离子液体是绿色化学领域的一个研究 热点 ${ }^{[1]}$. 离子液体凭借其独特的物化性质, 尤其是其 不挥发性且易于从反应体系中分离等优势, 被认为 是一种具有广泛应用前景的绿色溶剂及绿色催化 剂 ${ }^{[2]}$. 酸性离子液体具有取代工业酸催化材料的潜 力 ${ }^{[3]}$. 酸性离子液体主要包括氯铝酸咪唑离子液体 ${ }^{[3]}$ 、 单羧基咪唑离子液体 ${ }^{[4]}$ 以及单磺酸基咪唑离子液 体问等. 离子液体酸性强弱调节方式可以归纳为三
种: (1)改变氯铝酸咪唑离子液体阴离子中 $\mathrm{AlCl}_{3}$ 的 比例; (2)在阳离子结构中接人不同的酸性官能团(如 羧基、磺酸基等); (3)改变阳离子结构中碳链的长短. 酸性离子液体的酸性是其最重要的物性指标, 表征 离子液体酸性的手段主要有: 采用电位滴定法, 通过 $\mathrm{KOH}$ 标准溶液滴定, 测得离解常数 $\mathrm{p} K_{\mathrm{a}}$ 值 ${ }^{[}$; 用吡啶或 乙腈作为 FT-IR 谱探针测定 Brønsted/Lewis 酸性类 型[7]; 也可利用 UV-Vis 谱测定 Hammett 指数以表示

Received: July 28, 2005; Revised: September 8, 2005. *Correspondent, E-mail: cexhli@ scut. edu. cn; Tel/Fax: 020-87114707.

国家自然科学基金(20206010), 广东省自然科学基金 (031419)及广东省科技攻关(203C33503) 资助项目

C)Editorial office of Acta Physico-Chimica Sinica 
其酸强度 ${ }^{[8]}$.

以往功能化离子液体阳离子结构中的官能团通 常只有一个. 最近, 我们合成出多酯基和多羧基咪唑 离子液体, 并提出通过控制离子液体结构中接人羧 基的数量来调节离子液体酸性的新途径 ${ }^{[9-12]}$. 本文报 道多羊基咪唑离子液体的酸性表征结果, 并对其结 构与酸性的关联规律进行了分析.

\section{1 实验部分}

\section{1 试剂与仪器}

六氟磷酸 $\left(\mathrm{HPF}_{6}\right)$ 、四氟嗍酸 $\left(\mathrm{HBF}_{4}\right)$ 、三氟甲磺酸 $\left(\mathrm{CF}_{3} \mathrm{SO}_{3} \mathrm{H}\right)$ 以及三氟乙酸 $\left(\mathrm{CF}_{3} \mathrm{COOH}\right)$ 均为 Acros 产 品; 三乙胺、乙醇、二氯甲烷、盐酸、硝酸、硫酸以及 磷酸均为广州市金华大化学试剂有限公司提供的分 析纯试剂. 利用 EQUINOX-55 红外光谱仪(Brucker) 测量样品红外光谱, 溴化钾压片; AVANCE Digital (Brucker, $400 \mathrm{MHz}$ ) 核磁共振仪测量核磁共振谱, 内 标物为四甲基硅烷(TMS), 溶剂为DMSO-d6; ThermoFinnigan LCQ Deca XP 液质联用仪测得 ESI-MS 数 据; Elementar Vario EL 元素分析仪测得 C、H、N 元 素分析数据; METROM 758 KFD Titrino 微量水份 测定仪(容量法)测得离子液体中水的含量; PHS-25 型酸度计(上海精密科学仪器有限公司, 雷磁 E-201$\mathrm{C}$ 型 $\mathrm{pH}$ 复合电极)用于离子液体 $\mathrm{p} K_{\mathrm{a}}$ 值的测定.

\section{2 多羧基咪唑离子液体的合成 ${ }^{[10]}$}

多羧基咪唑离子液体 1a 1h、2a 2h 的结构式 如图 1 所示, 其制备过程如下.

首先, 根据文献[9-12]制备含有二酯基和三酯基 的咪唑离子液体. 然后, 取 $60 \mathrm{mmol}$ 的二酯基或三 酯基咪唑离子液体, 在闭合的反应装置中, 分别与 $20 \mathrm{~mL}$ 的盐酸在 $100{ }^{\circ} \mathrm{C}$ 下反应 $2 \mathrm{~h}$, 再旋转蒸发除去 溶剂, 并经真空干燥, 分别得到无色液态离子液体 $1 \mathrm{a}$ 和 $2 \mathrm{a}$.

在氮气保护下, 将 $60 \mathrm{mmol}$ 的离子液体 $\mathbf{1 a}$ 和 $2 \mathrm{a}$ 分别与 $66 \mathrm{mmol}$ 三乙胺在 $30 \mathrm{~mL}$ 二氯甲烷中混

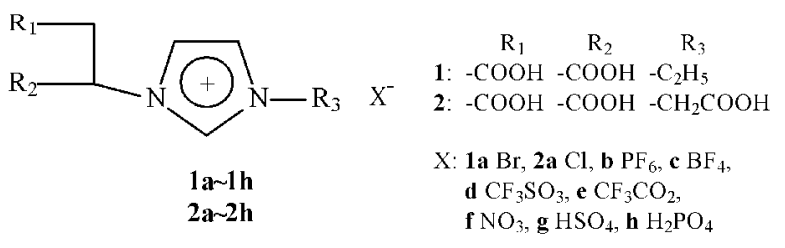

图 1 多羧基咪唑离子液体 1a 1h、2a 2h 的结构

Fig.1 Structures of polycarboxyl imidazolium ionic liquids 1a 1h、2a 2h
合, 室温搅拌 $24 \mathrm{~h}$; 将得到的白色固体按 $1: 1$ 的摩 尔比例分别与 $\mathrm{HPF}_{6} 、 \mathrm{HBF}_{4} 、 \mathrm{CF}_{3} \mathrm{SO}_{3} \mathrm{H} 、 \mathrm{CF}_{3} \mathrm{COOH}$ 、稀 硝酸、稀硫酸以及磷酸混合, 并在室温下摚拌 $24 \mathrm{~h}$, 旋转蒸发除去溶剂, 经真空干燥后, 得到离子液体 1b 1f $、 2 b \sim 2 f$.

\section{3 离子液体 $\mathrm{p} K_{\mathrm{a}}$ 值的测定}

多羧基咪唑离子液体的离解常数 $\mathrm{p} K_{\alpha}$ 值的测定 及计算过程参见文献 $[6,13]$. 配制 $0.01 \mathrm{~mol} \cdot \mathrm{L}^{-1}$ 的多 羧基咪唑离子液体水溶液; 在 $25{ }^{\circ} \mathrm{C}$ 下, 利用 0.100 $\mathrm{mol} \cdot \mathrm{L}^{-1} \mathrm{KOH}$ 标准溶液滴定. 同时, 利用该方法测量 乙酸和乙二酸的 $\mathrm{p} K_{\mathrm{a}}$ 值并与文献值 ${ }^{[14]}$ 相比较, 以确 认该方法是否准确.

\section{4 离子液体 Brønsted 酸性的确定 ${ }^{[]}$}

将吡啶与多羧基咪唑离子液体按 $1: 5$ (体积比)混 合均匀, 用 $\mathrm{KBr}$ 片液膜制样, 利用 Brucker EQUINOX 55 (分辨率 $1 \mathrm{~cm}^{-1}$ ) 测定红外光谱.

\section{2 结果与讨论}

\section{1 多羧基咪唑离子液体的合成}

多羧基咪唑离子液体 1a 1h、2a 2h 的结构和 纯度都通过 ${ }^{1} \mathrm{H} N M R 、{ }^{13} \mathrm{C}$ NMR 、ESI-MS、FT-IR、C、 $\mathrm{H} 、 \mathrm{~N}$ 元素分析以及水含量测试等检测手段确认.

\section{2 多羧基咪唑离子液体的 $\mathrm{p} K_{\mathrm{a}}$ 值的测定}

乙酸 (4.76) 和乙二酸 $(1.23)$ 的 $\mathrm{p} K_{\mathrm{a}}$ 测量值与文献 值 ${ }^{[14]}$ 一致, 说明所用方法及操作是准确、可信的.

多羧基咪唑离子液体 1a 1 $1 \mathrm{~h} 、 2 \mathrm{a} \sim 2 \mathrm{~h}$ 的 $\mathrm{p} K_{\mathrm{a}}$ 值 测量结果如图 2 所示, 由图 2 中可见, 1a 1h 的 $\mathrm{p} K_{\mathrm{a}}$ 分别为 $1.78 、 1.66 、 1.89 、 1.92 、 1.87 、 1.64 、 1.58 、 1.69$; $2 \mathrm{a} \sim 2 \mathrm{~h}$ 的 $\mathrm{p} K_{\mathrm{a}}$ 分别为 $1.61 、 1.53 、 1.73 、 1.76 、 1.70$ 、 $1.49 、 1.43 、 1.55$. 多羧基咪唑离子液体 $\mathrm{p} K_{\mathrm{a}}$ 值在 $1.43 \sim$ 1.92 范围内. 有研究表明, 离子液体的 $\mathrm{p} K_{\mathrm{a}}$ 值越小,

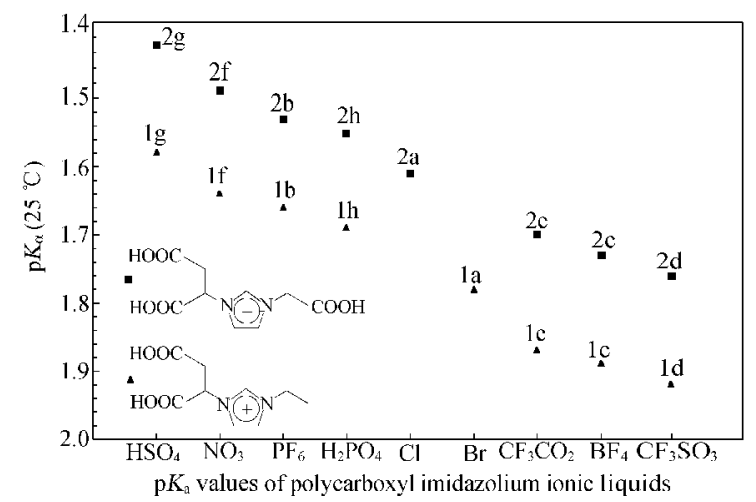

图 $225^{\circ} \mathrm{C}$ 时多羧基咪唑离子液体 $1 \mathrm{a} \sim 1 \mathrm{~h} 、 2 \mathrm{a} \sim 2 \mathrm{~h}$ 的 $\mathrm{p} K_{\mathrm{a}}$ 值

Fig.2 $\mathrm{p} K_{\mathrm{a}}$ values of polycarboxyl imidazolium ionic liquids (IL)1a 1h, 2a 2h at $25{ }^{\circ} \mathrm{C}$ 
其酸性就越强 ${ }^{6}$. 说明所合成的系列多羧基咪唑离 子液体具有中等强度酸性.

三羧基咪唑离子液体 $\mathbf{2 a} 2 \mathrm{~h}$ 的 $\mathrm{p} K_{\mathrm{a}}$ 值（1.43 1.76)比含有相同阴离子的二羧基咪唑离子液体 1a $1 \mathrm{~h}$ 的 $\mathrm{p} K_{\mathrm{a}}$ 值(1.58 1.92)小, 结合单羧基咪唑离子液 体的 $\mathrm{p} K_{\mathrm{a}}$ 值 $(1.90 \sim 4.11)^{[6]}$, 可以认为, 阳离子中羧基 数目越多, 离子液体的酸性越强.

通过测量羧酸的离解常数 $\mathrm{p} K_{\mathrm{a}}$ 值, 可以估算羧 酸中取代基诱导效应的强弱 ${ }^{[15]}$. 首先, 多羧基咪唑离 子液体 $1 \mathrm{a} \sim 1 \mathbf{h} 、 \mathbf{2 a} \sim 2 \mathrm{~h}$ 的 $\mathrm{p} K_{\mathrm{a}}$ 值(1.43 1.92)比丁二 酸(4.22)小得多. 这是由于咪唑离子液体结构中, 阳离 子咪唑环吸电子的诱导效应使得电子沿着碳链向咪 唑环偏移, 导致羧基负离子的负电荷分散且趋于稳 定, 羧基中的氢更容易离解, 从而增强离子液体的酸 性. 其次, 饱和直链二元羧酸结构中 ${ }^{[15]}$, 羧基有强 的吸电子效应, 能对另一个羧基的离解产生影响, 两个羧基越近, 烷基链越短, 这种影响就越大. 但随 着烷基链的增长, 烷基的超共轭效应通常会减弱饱 和直链二元羧酸的酸性 $\left(\mathrm{p} K_{\mathrm{a}}\right.$ 值 ${ }^{[14]}$ : 乙二酸 1.23 ; 丙二 酸 2.83; 丁二酸 4.22 ; 戊二酸 4.34 ; 已二酸 4.43). 多 羧基咪唑离子液体 $\mathbf{1 a} \sim \mathbf{1 h} 、 \mathbf{2 a} \sim \mathbf{2 h}$ 的 $\mathrm{p} K_{\mathrm{a}}$ 值（1.43 1.92)接近于乙二酸 (1.23), 说明咪唑环吸电子的诱导 效应弱于且接近于羧基强吸电子的诱导效应, 可以 部分抵消烷基链超共轭效应的影响. 有报道表明, 单 乙酸基咪唑离子液体的 $\mathrm{p} K_{\mathrm{a}}$ 值(1.90 2.03) 比卤代乙 酸 $\left(\mathrm{XCH}_{2} \mathrm{COOH}, \mathrm{X}=\mathrm{F} 、 \mathrm{Cl} 、 \mathrm{Br} 、 \mathrm{I}\right)$ 的 $\mathrm{p} K_{\mathrm{a}}$ 值 $(2.66 \sim 3.13)$ 小问, 也说明了带正电荷的咪唑环的吸电子诱导效应 很强, 甚至比卤素原子更强. 并且, 单羧基离子液体 的单晶衍射结果表明 [ , 羧基对咪唑环的结构(如键 长、键角)没有明显的影响.

阴离子对离子液体的酸性也有一定的影响. 阳 离子相同、阴离子不同的多羧基咪唑离子液体酸性 强弱顺序为: $\mathrm{HSO}_{4}^{-}>\mathrm{NO}_{3}^{-}>\mathrm{PF}_{6}^{-}>\mathrm{H}_{2} \mathrm{PO}_{4}^{-}>\mathrm{Cl}^{-} 、 \mathrm{Br}^{-}>$ $\mathrm{CF}_{3} \mathrm{CO}_{2}^{-}>\mathrm{BF}_{4}^{-}>\mathrm{CF}_{3} \mathrm{SO}_{3}^{-}$. 阴离子为无机含氧酸根的 离子液体比阴离子为有机含氧酸根的离子液体酸性 更强. 这主要是不同阴离子与阳离子中羧基形成氢 键能力的差异所导致 ${ }^{[16]}$.

\section{3 多羧基咪唑离子液体 Brønsted 酸性的确定}

研究表明 ${ }^{[7]}$, 吡啶与离子液体作用后, 在 1540 $\mathrm{cm}^{-1}$ 附近出现的吸收带指示 Brønsted 酸性, 在 1450 $\mathrm{cm}^{-1}$ 附近出现的吸收带指示 Lewis 酸性.

如图 3 所示, 多羧基咪唑离子液体 1a 1h、2a

2h 与吡啶作用后的 FT-IR 谱图上都只是在 1540
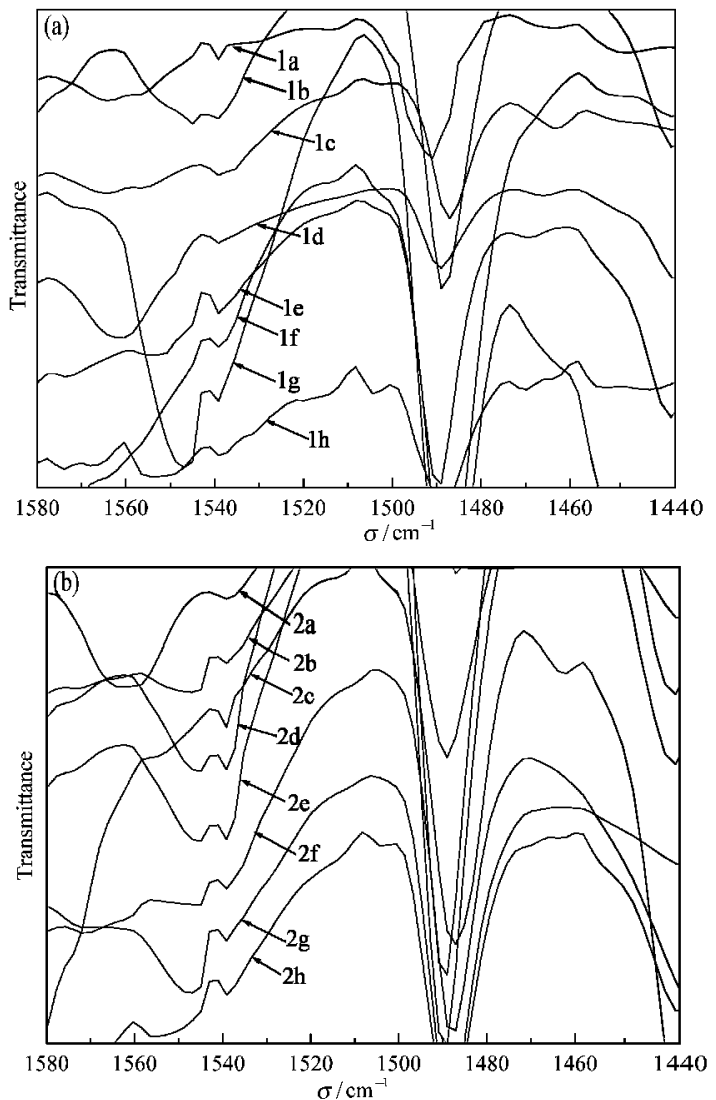

图 3 以吡啶为探针的多羧基咪唑离子液体 $1 \mathrm{a} \sim 1 \mathrm{~h}$ (a)、2a 2h (b) 的红外光谱

Fig.3 FT-IR spectra of polycarboxyl imidazolium ionic liquids using pyridine as probe

(a) 1a 1h; (b) 2a 2h

$\mathrm{cm}^{-1}$ 附近出现新的吸收带. 这说明多羧基咪唑离子 液体只具有 Brønsted 酸性, 与这些离子液体的结构 特征一致。

\section{3 结 论}

多羧基咪唑离子液体只具有Brønsted酸性, 其 $\mathrm{p} K_{\mathrm{a}}$ 值在1.43 1.92范围内, 由于咪唑环的强吸电子诱 导效应, 决定了此类离子液体具有中等强度的酸性. 通过改变离子液体咪唑环上取代羧基的数目并与不 同阴离子组合, 可达到调节离子液体酸性强弱的目 的.

\section{References}

1 Seddon, K. R. Nature Materials, 2003, 2: 363

2 Zhao, D. B.; Wu, M.; Kou, Y.; Min, E. Z. Catal. Today, 2002, 74 (1-2): 157

3 Yang, Y. L.; Wang, X. H.; Kou, Y. Chin. J. Catal., 2004, 25(1): 60 [杨雅立, 王晓化, 寇 元. 催化学报(Cuihиа Xиеbao), 2004, 25 (1): 60] 
4 Fraga-Dubreuil, J.; Bazureau, J. P. Tetrahedron Lett., 2000, 41 (38): 7351

5 Cole, A. C.; Jensen, J. L.; Ntai, I.; Tran, K. L. T.; Weaver, K. J.; Forbes, D. C.; Davis, Jr. J. H. J. Am. Chem. Soc., 2002, 124(21): 5962

6 Fei, Z. F.; Zhao, D. B.; Geldbach, T. J.; Scopelliti, R.; Dyson, P. J. Chem. -Eur. J., 2004, 10(19): 4886

7 Yang, Y. L.; Kou, Y. Chem. Commun., 2004(2): 226

8 Thomazeau, C.; Olivier-Bourbigou, H.; Magna, L.; Luts, S.; Gilbert, B. J. Am. Chem. Soc., 2003, 125(18): 5264

9 Geng, W. G.; Li, X. H.; Wang, L. F.; Pan, W. P.; Duan, H. L. Synthesis of multiester-appended imidazolium task-specific ionic liquids. In: Seddon, K. R. ed. 1st. International congress on ionic liquids (COIL). Frankfurt: Dechema, 2005: 318 319

10 Geng, W. G.; Li, X. H.; Duan, H. L.; Pan, W. P. Chin. Chem. Lett., 2006, 17(2): 169

11 Li, X. H.; Geng, W. G.; Pan, W. P.; Duan, H. L. Synthesis of 1-diester-3-alkylimidazolium ionic liquids. CN 10032 669.X. 2005
[李雪辉, 耿卫国, 潘微平, 段红丽. 1-双酯基-3-烷基咪唑离子液 体及其制备方法. 中国专利, 10032 669.X 2005]

12 Li, X. H.; Geng, W. G.; Pan, W. P.; Duan, H. L. Synthesis of 1, 3diesterimidazolium ionic liquids. CN 10032 681. 0.2005 [李雪 辉, 耿卫国, 潘微平, 段红丽. 1,3-二酯基取代咪唑型离子液体及 其制备方法. 中国专利, 10032681.0 .2005$]$

13 Albert, A.; Serjeant, E. P. The determination of ionization contants: a laboratory manual. 3rd. ed. New York: Chapman and Hall, 1984: 25

14 Stark, J. G.; Wallace, H. G. Chemsitry data book. Transe Yang, H. C. Beijing: Petrochemical Industry Press, 1980: 113 [化学数据手 册. 杨厚昌译. 北京: 石油工业出版社, 1980: 113]

15 Xing, Q. Y.; Xu, R. Q.; Zhou, Z. Fundamental organic chemistry(I). Beijing: People Education Press, 1980: 420 [邢其毅, 徐瑞秋, 周 政. 基础有机化学.上册. 北京: 人民教育出版社, 1980: 420]

16 Wasserscheid, P.; Keim, W. Angew. Chem. Int. Ed., 2000, 39(21): 3772 